\title{
Percolation on a non-homogeneous Poisson blob process
}

\author{
Fabio P. Machado ${ }^{\dagger}$ \\ Instituto de Matemática e Estatística, Universidade de São Paulo, Rua do Matão 1010, CEP 05508-090, São Paulo \\ SP, Brasil. $\quad$ fmachadodime.usp.br
}

We present the main results of a study for the existence of vacant and occupied unbounded connected components in a non-homogeneous Poisson blob process. The method used in the proofs is a multi-scale percolation comparison.

Keywords: Poisson blob model, continuum percolation, phase transition, multi-scale percolation

\section{Introduction}

One of the most well known examples of phenomena that introduces and motivates the study of continuum percolation is the process of the ground getting wet during a period of rain. At each point hit by a raindrop, one sees a circular wet patch. Right after the rain begins to fall what one sees is a small wet region inside a large dry region. At some instant, so many raindrops have hit the ground that the situation changes from that to a small dry region inside a large wet region. Typically, the parameter in which there is a phase transition behaviour is the density of the raindrops.

Continuum percolation models in which each point of a two-dimensional homogeneous Poisson point process is the centre of a disk of given (or random) radius $r$, have been extensively studied. In this note we present phase transition results for a sequence of Poisson point process which defines Poisson Boolean models and whose rates depend on the past. In order to prove our results we rely on a multiscale percolation structure. General reference for percolation and continuum percolation are the books of Grimmett [2] and Meester and Roy [3]. A nice example of the use of multi-scale percolation technique can be found is Fontes et al [1].

\section{Model and phase transition results}

Let $\beta>0$ be fixed number. Define $A_{0}=\emptyset$. Having defined the sets $A_{0}, A_{1}, \ldots, A_{n}$, define the process $X_{n+1}$ as the non-homogeneous Poisson point process with intensity function given by:

$$
f_{n+1}(x)=\exp \left(-\beta\left|B(x, n+1) \backslash \cup_{k=0}^{n} A_{k}\right|\right)
$$

where $B(a, r)$ is the square of length $r$ having centre at $a$ and $|C|$ is the area (Lebesgue measure) of the set $C$.

\footnotetext{
${ }^{\dagger}$ The author is thankful to CNPq (300226/97-7) for fi nancial support. 
Let $\left\{x_{i}^{(n+1)}: i \geq 1\right\}$ be the set of points from the process $X_{n+1}$. Define the set $A_{n+1}=\cup_{i=1}^{\infty} B\left(x_{i}^{(n+1)}, n+1\right)$ as the random set covered by the boxes from the process $X_{n+1}$. Define the total covered set $A_{\infty}=\bigcup_{n=0}^{\infty} A_{n}$.

The fundamental question in continuum percolation theory is about the existence of unbounded connected components. That is why we ask the following questions about the random set $A_{\infty}$ and its complement, the set $A_{\infty}^{c}$. Let $A$ be the component of $A_{\infty}$ which contains the origin. If the origin is not contained in $A_{\infty}$, this is the empty set. Define

$$
\theta(\beta)=\mathbb{P}_{\beta}(A \text { is unbounded }) .
$$

It is clear that $\theta(\beta)$ is a decreasing function of $\beta$. Hence define the critical parameter $\beta_{c}$ as follows:

$$
\beta_{c}=\sup \{\beta>0: \theta(\beta)>0\} .
$$

The following result holds

\section{Theorem 1.}

$$
0<\beta_{c}<\infty .
$$

Similar questions can also be asked the complement set of $A_{\infty}$. Define $C$ as the component of $\left(A_{\infty}\right)^{c}$ which contain the origin. Define the vacant percolation probability as

$$
\theta^{\star}(\beta)=\mathbb{P}_{\beta}(C \text { is unbounded }) .
$$

In this case, we have that $\theta^{\star}(\beta)$ is an increasing function of $\beta$. Hence define the critical parameter $\beta_{c}^{\star}$ as follows:

$$
\beta_{c}^{\star}=\inf \left\{\beta>0: \theta^{\star}(\beta)>0\right\} .
$$

We also prove the following theorem

\section{Theorem 2.}

$$
0<\beta_{c}^{\star}<\infty
$$

It is clear that $X_{1}$ is actually an homogeneous Poisson process with intensity $\exp (-\beta|B(0,1)|)$. Thus, $A_{1}$ will contain the covered set of a Poisson Boolean model with radius random variable being degenerate at $1 / 2$ and intensity $\exp (-\beta|B(0,1)|)$. Thus, if $\exp (-\beta|B(0,1)|)>\lambda_{c}$, the probability that the origin is contained in an unbounded component of $A_{1}$ is positive, where $\lambda_{c}$ is the critical intensity of the Poisson Boolean model with radius being degenerate at $1 / 2$. Therefore, we have $\theta(\beta)>0$ for this $\beta$. Hence, we have that $\beta_{c}>0$. A similar argument also holds for $\beta_{c}^{\star}$ and we can easily show that, $\beta_{c}^{\star}>0$.

This is an announcement of results from a joint work with P. Ferrari, L. Fontes, S. Popov and A. Sarkar. The proofs rely on a multi-scale comparison argument to prove that the probability of certain events related to the existence of an unbounded connected component is exponentially close to 1 for large values of $\beta$.

\section{References}

[1] Fontes, L. R.; Schonmann, R. H. And Sidoravicius, V. (2002) Stretched exponential fixation in stochastic Ising models at zero temperature. Comm. Math. Phys. 228, no. 3, 495-518.

[2] Grimmett, G. (1999). Percolation, Second Edition. Springer, New York.

[3] Meester, R. And Roy, R. (1996). Continuum Percolation. Cambridge University Press, Cambridge. 